\title{
Clinical management of extrapleural septal solitary fibrous tumor: A case report
}

\author{
MICHELE CAVALIERE $^{1}$, EVA PIRO ${ }^{1}$, MASSIMO MASCOLO $^{2}$ CLAUDIO DI NOLA $^{1}$, SALVATORE ALLOSSO ${ }^{1}$, \\ ANTONELLA M.DI LULLO ${ }^{1}$, GAETANO MOTTA ${ }^{3}$, MAURIZIO IENGO ${ }^{1}$ and ELENA CANTONE ${ }^{1}$ \\ ${ }^{1}$ Department of Neuroscience, Reproductive and Odontostomatological Sciences-ENT Section; \\ ${ }^{2}$ Department of Advanced Biomedical Sciences, Pathology Unit, University of Naples 'Federico II'; \\ ${ }^{3}$ Otorhinolaryngology, Head and Neck Surgery Unit, Department of Mental and Physical Health and Preventive Medicine, \\ Università degli Studi della Campania Luigi Vanvitelli, I-80131 Naples, Italy
}

Received January 21, 2020; Accepted May 19, 2020

DOI: $10.3892 / \mathrm{ol} .2020 .11789$

\begin{abstract}
Solitary fibrous tumor (SFT) is a rare neoplasm arising from the pleura, although it can sometimes affect extrapleural sites, including the head and neck. The sinonasal involvement is exceptional. Recent literature defines the SFT as a single spectrum of mesenchymal tumors, including hemangiopericytoma, which is currently considered a cellular phenotypic variant. The current case describes a rare case of a nasal septal SFT. The mass was embolized and then removed through endonasal endoscopic surgery. Histopathologic examination demonstrated positive immunoreactivity for CD34, and negative for keratin and S100 protein confirming the diagnosis of benign nasal SFT. After two years of follow-up, no relapse was observed. Diagnostic assessment of SFT requires nasal endoscopy, imaging and histopathological examination and a long time follow up is mandatory.
\end{abstract}

\section{Introduction}

Solitary fibrous tumor (SFT) is a rare neoplasm that generally arises from the pleura or, more rarely, from other serosal membranes. The literature reports different extrapleural sites, which supports the hypothesis of a submesenchymal origin instead of a mesothelial origin (1-4).

Extrapleural SFT are fibroblastic/myofibroblastic neoplasms, rarely metastasizing and with intermediate biological behavior (5). The wide heterogeneity of denominations used for this neoplasm is simply the representation of its much-disputed histogenesis; indeed, various benign and malignant tumors may share the same growth pattern. The

Correspondence to: Dr Elena Cantone, Department of Neuroscience, Reproductive and Odontostomatological Sciences-ENT Section, University of Naples 'Federico II', via Pansini 5, I-80131 Naples, Italy E-mail: elena.cantone@unina.it; cantoneent@gmail.com

Key words: solitary fibrous tumor, head and neck, hemangiopericytoma, mesenchymal tumor, extrapleural tumor haemangiopericytoma (HPC)-like features are common to three categories of neoplasms: Non-HPC neoplasms (e.g., synovial sarcoma), occasionally displaying HPC-like features; true HPCs (glomangiopericytoma/myopericytoma, infantile myofibromatosis) with evident myoid-pericytic differentiation; and the SFT group (giant cell angiofibromas, lipomatous HPCs) (6). The World Health Organization (WHO) considered HPC as histological variant of SFT and no longer as separate entities, due to recent findings about its immunohistochemical and molecular features. Hence, according to WHO, HPC is currently a cellular phenotypic variant of SFT that is a single spectrum of mesenchymal tumors. These fibroblastic lesions, usually pertain to adults and can occur at any site (7).

Although the etiology of SFT remains still undisclosed, the pathogenesis is related to the NAB2-STAT6 gene fusion caused by paracentric inversion on chromosome $12 \mathrm{q} 13$. NAB2-STAT6 gene encodes a chimeric protein which activates ERG1 and seems to deregulate ERG1-dependent target genes $(4,5,7)$.

From clinical point of view, SFT is a slow-growing mass in the fifth and sixth decades, with no sex predilection and may be responsible for paraneoplastic syndromes and in particular, for hypoglycemia probably caused by the production of an insulin-like growth factor (6). Although most SFTs have a good prognosis, about $10-40 \%$ of cases relapse or metastasize $(3,4,8)$. For instance, the clinically aggressive behavior is related to pathological criteria of malignancy. However, a clear correlation between hystology and clinical behavior has not been identified yet. Furthermore, considering its rarity, there are no guidelines and the course of SFT remains still unpredictable $(3,4,8)$.

SFT has been described in a wide variety of atypical extrapleural sites of mesenchymal origin, among which head and neck regions, para-pharyngeal space, tongue, larynx, and parotid gland. The presence of SFTs in the sino-nasal cavities are unusual; indeed, very few cases are described in the literature (3). Immunohistochemical pattern is useful to differentiate SFT from other soft tissue tumors (9-11). In particular, these lesions have 2 main characteristics, the solid spindle and the diffuse sclerosing types, usually within the same lesion. Though chemo-radiotherapy have been used to control the 
tumor, surgery remains the treatment of choice (12). We report a very rare case of a large extrapleural SFT of the nasal septum undergoing endoscopic surgery.

\section{Case report}

A 55-year-old man presented at ENT Unit of 'Federico II' University complaining right-sided nasal obstruction, discharge and recurrent epistaxis for 2-years. He reported a history of cigarette smoking, hypertension, diabetes mellitus type II, hypercholesterolemia, and previous iliac artery percutaneous transluminal angioplasty and stenting.

There was no palpable latero-cervical lymphadenopathy and the ultrasound of the neck was negative. Nasal endoscopy revealed a rough reddish bleeding mass with implantation point on the septum occupying the right nasal fossa. Computed Tomography (CT) scan confirmed the presence of the right nasal mass without invading the anterior cranial fossa, signs of bone destruction and remodeling, or distant metastases. Magnetic resonance imaging (MRI) showed a lesion in the right nasal fossa with a hypointense signal on T1-weighted images and hyperintense $\mathrm{T} 2$-weighted right-sided nasal mass (Fig. 1) (13). The neoplasm showed a prominent and non-homogeneous enhancement after gadolinium injection.

Due to the abundant vascularization of the lesion revealed by imaging, angiographic study was performed. It showed an arterial supply from the anterior ethmoidal artery, so a selective embolization was performed.

A diagnostic biopsy of the mass was carried out under local anesthesia. Histologic investigation revealed a low-grade mesenchymal neoplasm, morphologically and immunophenotypically accordant with the diagnosis of nasal SFT. Subsequently, the patient underwent surgery under general anesthesia to remove the lesion, endonasal endoscopic approach was performed. The insertion of tumor on the right anterior-superior region of the nasal septum was identified and the mass was completely removed, achieving a mucosal flap with sub-muchoperichondrium incision.

Histology revealed a homogeneous and diffuse proliferation of spindle cells that alternated hypercellular and less cellular areas with collagenized stroma, characterized by mild pleomorphism and more than occasional mitoses; necrosis was absent. The tumour showed a prominent vascular network consisting of branching vessels with a slightly thickened wall. The immunohistochemistry showed positivity of neoplastic cells for vimentin, CD34 and CD99 and negativity for S100 protein, desmin, pan-cytokeratin, smooth muscle actin and LCA. The proliferation index Ki-67 was less than 5\%. The morphology and immunophenotype strongly suggested a solitary fibrous tumour (Fig. 2).

The postoperative course did not show complications and the patient was discharged within $24 \mathrm{~h}$. After 2 years of follow-up, no relapse was observed at endoscopy and imaging studies.

\section{Discussion}

SFT is a slow-growing, well-encapsulated spindle cell tumor resembling fibro-sarcoma. However, unlike fibrosarcoma, SFT did not show any sign of metastasis or infiltration.

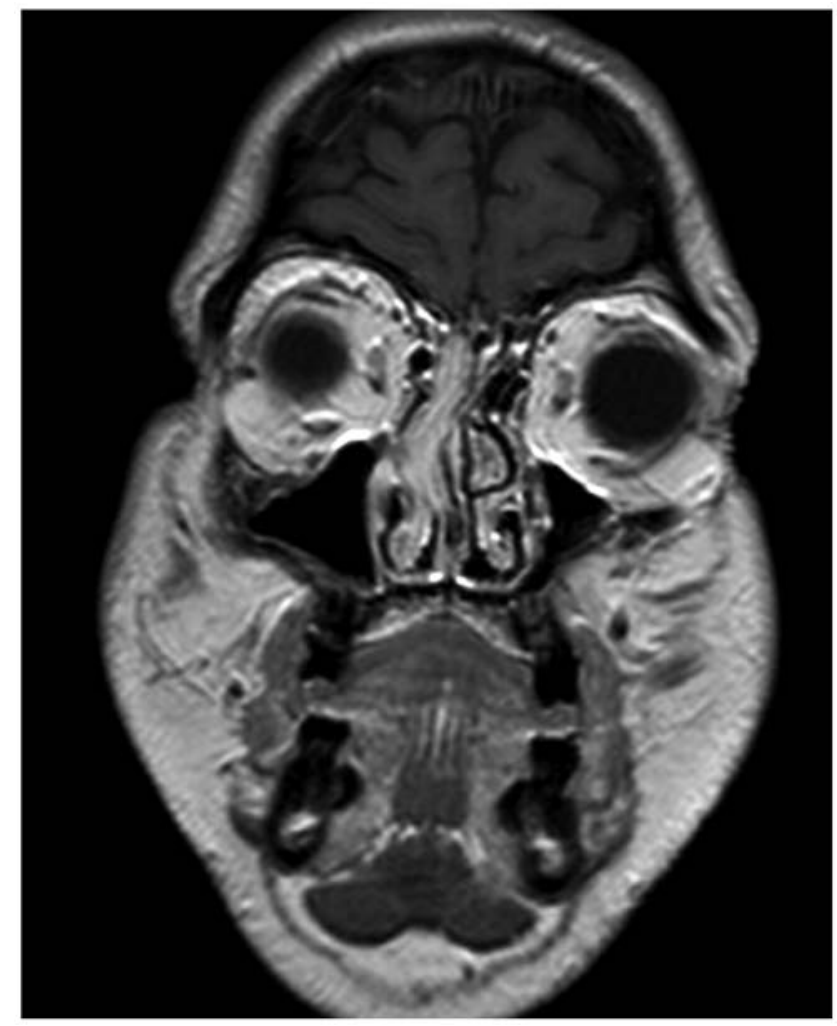

Figure 1. Preoperative MRI. The MRI showed a lesion in the right nasal fossa with a hypointense signal on T1-weighted and hyperintense signal on T2-weighted images.

Increasing evidence suggested a mesenchymal origin of these tumors (1). Extrapleural localization (including the oral cavity and orbit) is rare and head and neck involvement is exceptional, approximately $5-27 \%$ (3). SFT rarely involves the nasal fossa and the paranasal sinuses, so SFT is sometimes not distinguishable from other mesenchymal lesions (3,9-11). To our knowledge, a limited number of SFT involving sino-nasal cavities have been described. A review of English literature has identified about 92 cases of sino-nasal area, most of them provided by single case report (3). Males and females are affected equally. Diagnosis of sino-nasal SFT is based on nasal endoscopy and imaging, $\mathrm{CT}$ assesses tumor extension and bone resorption, whereas MRI assesses orbit or endocranial extension. Particularly, CT shows a well-delineate isodense mass with heterogeneous contrast enhancement, whereas MR shows a hypo- or isointense on T1-weighted mass and hypo- or, hyperintense on T2-weighted mass, with heterogeneous contrast enhancement after contrast (13). The gold standard of therapy is surgery, endoscopic sinus surgery with elevation and resection of the periosteum of the tumor-contacting bone allows a complete resection. Preoperative embolization of the mass may decrease bleeding, although some authors reported rare inta/post-operative bleeding (14). Additional therapeutic options are sole radiotherapy, chemotherapy or embolization (14). SFTs are capsulated tumors, composed of cytologically bland spindle cells set in a collagenous stroma. Immunohistochemical analysis shows cells positive for $\mathrm{CD} 34$, vimentin and frequently bcl-2, and negative for keratin, desmin and S100 protein $(1,3,5,15)$. 
A
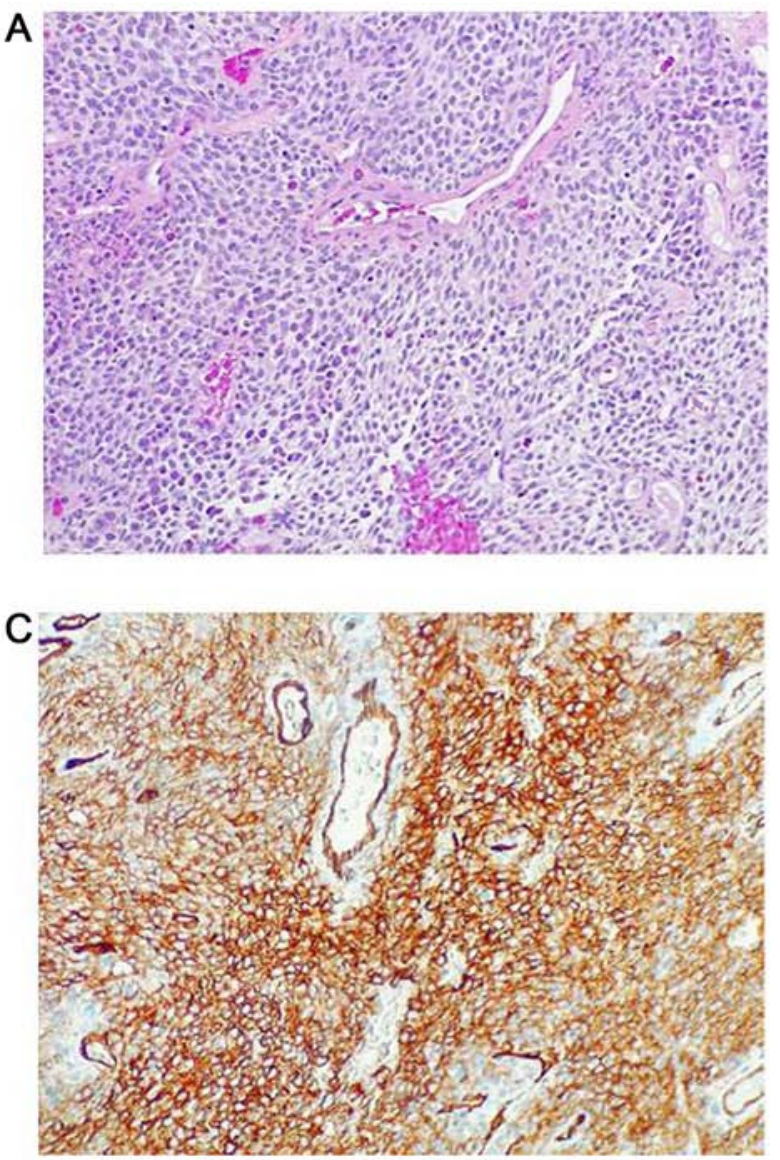

B

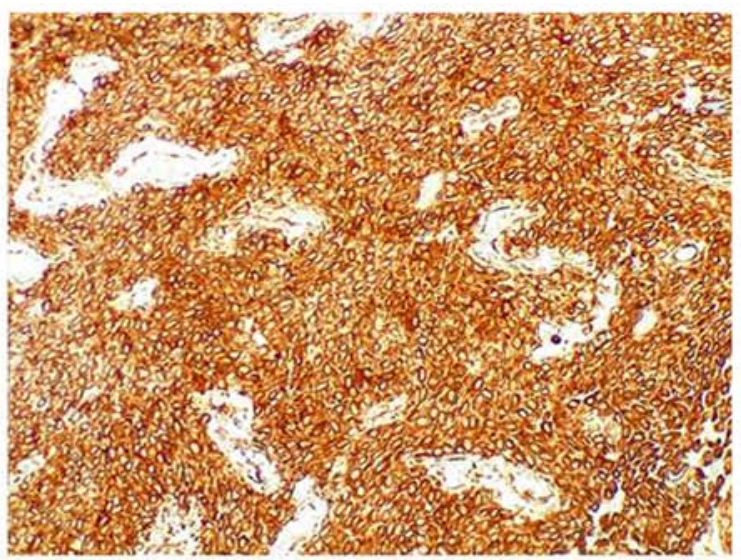

D

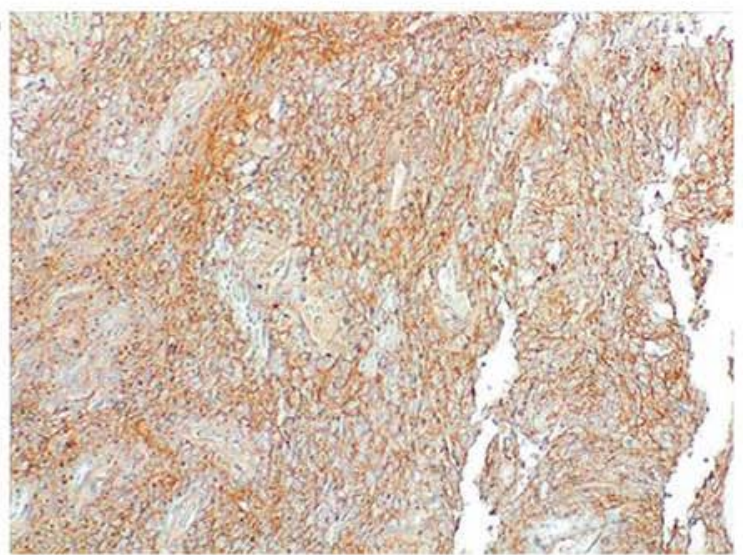

Figure 2. Immunohistochemistry. (A) A diffuse proliferation composed of monotonous spindle cells with vesicular nuclei and abundant cytoplasm and branching vessels (haematoxylin and eosin, original magnification x200); (B) Neoplastic cells exhibited positivity for vimentin (vimentin, original magnification x200); (C) Positivity of neoplastic cells for CD34 (anti-CD34, original magnification x400); (D) Neoplastic cells expressed CD99 (anti-CD99, original magnification $\mathrm{x} 200)$. $\mathrm{CD}$, cluster of differentiation

Disease progression, relapse or metastasis, can be rarely found. Pathologic criteria for malignancy are not well determinated. The WHO classifies SFT as malignant based on the presence of hypercellularity, increased mitoses ( $>4$ mitoses per 10 high power fields), cytological atypia, tumor necrosis, and/or infiltrative margins (3). For instance, histo-pathology seems to be an imperfect predictor of clinical behavior. Precisely, some tumors with morphologic parameters suggestive of malignancy would behave as benign mass. Recurrence and metastases may lack atypical histologic features (3).

In addition, other models considering a combination of various patient characteristics as age ( $\geq 55$ years), tumor size $(>10$ or $\geq 15 \mathrm{~cm}$ ), and mitotic index ( $>4$ mitoses per 10 high power fields), or tumor characteristics as mitotic rate, cellularity, and pleomorphism have been proposed as behavior predictor of SFTs (3). Recent studies point out the predictive role of telomerase reverse transcriptase (TERT) promoter mutations as molecular marker of prognosis in SFT high-risk patients. The combination of TERT promoter mutations and other clinicopathologic risk features might improve the accuracy of predicting outcomes in patients with SFT (7).

In this report we presented a very rare case of SFT involving the nasal septum undergoing surgical endoscopy and disease free after 24 months follow up.

Although previous authors reported clinical cases of nasal SFT, these cases did not present an exclusive nasal septal localization and did not perform embolization before endoscopic surgery. Other surgeons performed an open surgical technique. Furthermore, in the report of Zielinska-Kazmierska and in the report of Chauhan the patient did not perform MRI (16-18).

Our patient reported a 2 years history of symptoms in line with literature, in which the symptoms before diagnosis ranged from 25 days to 24 months, with an average of 9.9 months (3).

Interestingly, some of these tumors are recognized to be associated with systemic hypoglycemia (6).

Unlikely, our patient was suffering from diabetes, therefore it could be hypothesized that the disease was, as well, related to impaired glucose metabolism or we can speculate that therapy with hypoglycemic agents could have caused hypoglycemia as risk factor for STF.

In conclusion, in our opinion the diagnosis of sino-nasal STF is challenging and the prognosis is even more demanding. We, therefore, proposed integrating the assessment of TERT promoter mutational status to improve risk prediction in patients with sino-nasal STF, in addition to nasal endoscopy, imaging and histopathological examination. We think that a careful evaluation of blood glucose levels is also helpful.

Furthermore, in our experience, preoperative embolization has been useful not only to optimize the surgical resection but also as additional therapeutic option per se, although many authors do not consider it necessary for the purpose of controlling intraoperative bleeding (14). 


\section{Acknowledgements}

Not applicable.

\section{Funding}

No funding was received.

\section{Availability of data and materials}

The datasets used and/or analyzed during the present study are available from the corresponding author on reasonable request.

\section{Authors' contributions}

EC conceived and designed this case report. MC, EP, CDN and $\mathrm{EC}$ wrote the initial draft of the report. EP, MC, GM, MM and CDN collected data and wrote the initial draft. AMDL, SA and MI acquired the data in the diagnostic imaging. MM acquired staining and histological images. All authors have read and approved the final version of the manuscript.

\section{Ethics approval and consent to participate}

Written informed consent for surgery was obtained from the patient.

\section{Patient consent for publication}

Written informed consent for publication of the present report was obtained from the patient.

\section{Competing interests}

The authors declare that they have no competing interests.

\section{References}

1. Yalcin CE and Tihan T: Solitary fibrous tumor/hemangiopericytoma dichotomy revisited: A restless family of neoplasms in the CNS. Adv Anat Pathol 23: 104-111, 2016.

2. Ronchi A, Cozzolino I, Zito Marino F, Accardo M, Montella M, Panarese I, Roccuzzo G, Toni G, Franco R and De Chiara A: Extrapleural solitary fibrous tumor: A distinct entity from pleural solitary fibrous tumor. An update on clinical, molecular and diagnostic features. Ann Diagn Pathol 34: 142-150, 2018.
3. Thompson LDR and Lau SK: Sinonasal tract solitary fibrous tumor: A clinicopathologic study of six cases with a comprehensive review of the literature. Head Neck Pathol 12: 471-480, 2018

4. Demicco EG, Park MS, Araujo DM, Fox PS, Bassett RL, Pollock RE, Lazar AJ and Wang WL: Solitary fibrous tumor: A clinicopathological study of 110 cases and proposed risk assessment model. Mod Pathol 25: 1298-1306, 2012.

5. Robinson DR, Wu YM, Kalyana-Sundaram S, Cao X, Lonigro RJ, Sung YS, Chen CL, Zhang L, Wang R, Su F, et al: Identification of recurrent NAB2-STAT6 gene fusions in solitary fibrous tumor by integrative sequencing. Nat Genet 45: 180-185, 2013.

6. Gengler $\mathrm{C}$ and Guillou L: Solitary fibrous tumour and haemangiopericytoma: Evolution of a concept. Histopathology 48: 63-74, 2006.

7. Bahrami A, Lee S, Schaefer IM, Boland JM, Patton KT, Pounds S and Fletcher CD: TERT promoter mutations and prognosis in solitary fibrous tumor. Mod Pathol 29: 1511-1522, 2016.

8. Huang SC and Huang HY: Solitary fibrous tumor: An evolving and unifying entity with unsettled issues. Histol Histopathol 34: 313-314, 2019.

9. Cantone E, Borzillo V, Di Lullo AM, Marano L, Guadagno E, Mansueto G, Di Franco R, Cammarota F, Catalano L, Muto P and Iengo M: Cyberknife ${ }^{\circledR}$ system: A new therapeutic strategy for sinonasal solitary extramedullary plasmacytomae. J Biol Regul Homeost Agents 3: 763-768, 2017.

10. Cantone E, Di Lullo AM, Marano L, Guadagno E, Mansueto G, Capriglione P, Cammarota F, Catalano L and Iengo M: Strategy for the treatment and follow-up of sinonasal solitary extramedullary plasmacytoma: A case series. J Med Case Rep 11: 219, 2017.

11. Cantone E, Cavaliere M, Di Lullo AM, Guadagno E and Iengo M: Immunohistochemical patterns in the differential diagnosis of rhinopharyngeal granulocytic sarcoma. Oncol Lett 12: 2777-2781, 2016

12. Jurado-Ramos A, Ropero Romero F, Cantillo Baños E and Salas Molina J: Minimally invasive endoscopic techniques for treating large, benign processes of the nose, paranasal sinus, and pterygomaxillary and infratemporal fossae: Solitary fibrous tumour. J Laryngol Otol 123: 457-461, 2009.

13. Zhanlong M, Haibin S, Xiangshan F, Jiacheng S and Yicheng N: Variable solitary fibrous tumor locations: $\mathrm{CT}$ and MR imaging features. Medicine (Baltimore) 95: e3031, 2016.

14. Rizzo S, Giunta AAM and Pennacchi A: Sinonasal and rhinopharyngeal solitary fibrous tumour: A case report and review of the literature. Acta Otorhinolaryngol Ital 35: 455-458, 2015.

15. Lo Muzio L, Mascolo M, Capodiferro S, Favia G and Maiorano E: Solitary fibrous tumor of the oral cavity: The need for an extensive sampling for a correct diagnosis. J Oral Pathol Med 36: 538-42, 2007.

16. Chauhan SS, Krishnan J and Heffner DK: Solitary fibrous tumor of nasal cavity in patient with long-standing history of cocaine inhalation. Arch Pathol Lab Med 128: e1-e4, 2004.

17. Mathew GA, Ashish G, Tyagi AK, Chandrashekharan R and Paul RR: Solitary fibrous tumor of nasal cavity: A case report. Iran J Otorhinolaryngol 27: 307-312, 2015.

18. Zielińska-Kaźmierska B, Grodecka J and Szyszkowski A: Solitary fibrous tumor of the nasal cavity and paranasal sinuses: A case report. J Oral Biol Craniofac Res 5: 112-116, 2015. 